\title{
Antitumor Effect of Shikonin, a PKM2 Inhibitor, in Cholangiocarcinoma Cell Lines
}

\author{
UNCHALEE THONSRI ${ }^{1,2}$, WUNCHANA SEUBWAI ${ }^{2,3}$, SAKDA WARAASAWAPATI $^{4}$, SOPIT WONGKHAM ${ }^{1,2}$, \\ THIDARAT BOONMARS ${ }^{2,5}$, UBON CHA'ON ${ }^{1,2}$ and CHAISIRI WONGKHAM ${ }^{1,2}$ \\ ${ }^{1}$ Department of Biochemistry, Faculty of Medicine, Khon Kaen University, Khon Kaen, Thailand; \\ ${ }^{2}$ Cholangiocarcinoma Research Institute, Faculty of Medicine, Khon Kaen University, Khon Kaen, Thailand; \\ ${ }^{3}$ Department of Forensic Medicine, Faculty of Medicine, Khon Kaen University, Khon Kaen, Thailand; \\ ${ }^{4}$ Department of Pathology, Faculty of Medicine, Khon Kaen University, Khon Kaen, Thailand; \\ ${ }^{5}$ Department of Parasitology, Faculty of Medicine, Khon Kaen University, Khon Kaen, Thailand
}

\begin{abstract}
Background/Aim: Pyruvate kinase M2 (PKM2) is an enzyme that is predominantly overexpressed in various types of cancer. The role of PKM2 in liver fluke-associated cholangiocarcinoma (CCA) remains unclear. This study aimed to investigate the antitumor activity of shikonin, a PKM2 inhibitor, in CCA cells. Materials and Methods: Immunohistochemistry and immunoblotting were used to determine PKM2 expression in CCA tissues and cells. Antiproliferative effects of shikonin were evaluated using 3(4,5-dimethylthiazol-2-yl)-2,5-diphenyltetrazolium bromide, colony-formation and trypan blue exclusion assays. The antimetastatic activity of shikonin was determined using the Boyden chamber assay. Mechanisms by which shikonin inhibited CCA progression were determined. Results: PKM2 was overexpressed in CCA compared to normal bile duct epithelial cells. Shikonin significantly inhibited growth, and migration of CCA cells while inducing their death. A mechanistic study revealed that antitumor effects of shikonin in CCA cells depended on increased production of reactive oxygen species. Conclusion: Shikonin may be a novel therapeutic agent for patients with CCA.
\end{abstract}

Cholangiocarcinoma (CCA) is an aggressive malignancy which originates from bile duct epithelia. The highest incidence and mortality rate of CCA was found in Southeast

This article is freely accessible online.

Correspondence to: Assistant Professor Wunchana Seubwai, PhD, Faculty of Medicine, Khon Kaen University, Khon Kaen 40002, Thailand. Tel: +66 43202859, Fax: +66 43363708, e-mail: wunchanas@yahoo.com

Key Words: Cholangiocarcinoma, pyruvate kinase M2, shikonin, reactive oxygen species.
Asian countries, especially in northeastern Thailand (1). Recent epidemiological studies indicated that the incidence of CCA is different according to geographical risk factors and genetic backgrounds. Thamavit et al. showed that the infection with liver fluke, namely Opisthorchis viverrini (OV), is strongly associated with CCA development in Thailand, an area in which OV is endemic (2). Most patients with CCA present with an advanced stage of the disease, leading to a late diagnosis, poor prognosis and a short survival (3). Although surgical resection has remained a good choice for CCA therapy, only a very small number of cases can be operated on and this results in a high recurrence. At present, few neoadjuvant or adjuvant treatments for CCA are available as treatment options for patients with unresectable CCA or for after surgery. Chemoresistance and tumor relapse are still high in patients with CCA (4). Therefore, a new effective therapeutic agent for CCA is needed.

Metabolic reprogramming, first described by Hanahan and Weinberg, is a cancer hallmark (5). Most cancer cells prefer to use aerobic glycolysis than mitochondrial metabolism; this is known as the Warburg effect. Up-regulation of glycolytic enzymes have been reported in several cancer types including CCA, suggesting that these enzymes are potential targets for diagnosis and therapy of cancer (6). Pyruvate kinase M2 (PKM2), a key enzyme in the glycolytic pathway, is an isomeric form of pyruvate kinase, occurring in four isoforms: $\mathrm{R}, \mathrm{L}, \mathrm{M} 1$, and M2. PKM2 exhibits two major states, one is a highly active state in the tetrameric form and the other is the less active state in the dimeric form (7). Dimeric PKM2 facilitates the production of glycolytic intermediates for macromolecule biosynthesis and is frequently found in normal proliferating cells and cancer cells (8). In addition, the non-glycolytic function of PKM2 has also been reported. PKM2 is localized in the nuclear compartment where it activates oncogene expression by binding to the transcriptional coactivator of hypoxia-inducible factor 1 
(HIF1) and contributes to promoting the transactivation of HIF1 target genes. Nuclear PKM2 has also been reported to regulate transactivation of $\beta$-catenin that is involved in cellcycle progression. Moreover, based on protein kinase function, nuclear PKM2 phosphorylates include many signaling proteins such as the activator of transcription 3 (STAT3), histone H3, and BUB3 mitotic checkpoint protein, which are important in promoting tumorigenesis and cancer progression (9). Up-regulation of PKM2 expression has been reported in many cancer types such as colorectal (10), breast (11), and ovarian (12). Overexpression of PKM2 was found to be associated with poor outcome of patients with cancer. More evidence both in vitro and in vivo showed that loss of PKM2 significantly reduced aggressive features of cancer cells such as high proliferation, migration, invasion and chemoresistance, and induced apoptosis $(13,14)$. Therefore, PKM2 may have potential as a drug target for cancer therapy.

Promising anticancer activities of glycolytic inhibitors have been demonstrated both in vitro and in vivo (15). Of particular interest is shikonin, a traditional herbal medicinal that originates from plants containing naphthoquinone isolated from the root of Lithospermum erythrorhizon and has been identified as a specific PKM2 inhibitor. Shikonin has significant pharmacological effects including antimicrobial, anti-inflammatory, and antioxidant properties . Shikonin was recently identified as having anticancer activity against breast, gastric, and liver cancer (16). Inhibiting PKM2 by shikonin was reported to inhibit tumor growth, metastasis, and chemoresistance, inducing cell-cycle arrest and apoptosis $(17,18)$. The roles of PKM2 and the anticancer effect of shikonin on CCA cells remains unclear.

The present study aimed to investigate the impact of shikonin on cell proliferation, migration and death in CCA cell lines and an attempt was made to examine its potential mechanism by measurement of reactive oxygen species (ROS) levels. In addition, the role of PKM2 on tumorigenesis and progression of CCA were examined. PKM2 expression was determined in both hamster and human CCA tissues and its clinical significance was analyzed.

\section{Materials and Methods}

Cell culture and CCA tissues. Three CCA cell lines, KKU-055, KKU-100, and KKU-213A were established from primary tumors of patients with CCA. KKU-055 and KKU-100 were described as poorly differentiated adenocarcinoma (19). KKU-213A formed poorly differentiated squamous cell carcinoma (20). CCA cell lines were obtained from the Japanese Collection of Research Bioresources Cell Bank, Osaka, Japan. Cells were cultured in Hams F12 (Invitrogen, Carlsbad, CA, USA) supplemented with 10\% fetal bovine serum (Gibco/Invitrogen) and $1 \%$ penicillin-streptomycin (Gibco/Invitrogen) and incubated at $37^{\circ} \mathrm{C}$ with $5 \% \mathrm{CO}_{2}$.

Paraffin-embedded hamster CCA tissues $(n=60)$ were obtained from a liver fluke-associated hamster CCA model. Syrian golden hamsters were divided into four groups: Control untreated, OVinfected, $N$-nitrosodimethylamine (NDMA)-treated, and OV plus NDMA-treated as described elsewhere (21). Animals were sacrificed at 1,3 , or 6 months. The protocol of the study was approved by the Ethics Committee for Animal Research of Khon Kaen University (AEMDKKU1/2558).

Paraffin-embedded human CCA tissues $(n=80)$ were obtained from the specimen bank of the Cholangiocarcinoma Research Institute, Faculty of Medicine, Khon Kaen University, Thailand. All tissues were histologically proven to be CCA. Written informed consent was obtained from each patient and the protocol was reviewed and approved by the Ethics Committee for Human Research of Khon Kaen University (HE571283) based on the Declaration of Helsinki and ICH-Good Clinical Practice Guidelines.

Immunohistochemistry (IHC). PKM2 was detected in paraffinembedded CCA tissues using a standard immunohistochemistry protocol and IHC index as previously described (22). The CCA tissue slides were incubated with 1:600 rabbit antibody to PKM2 (Cell Signaling Technology, Boston, MA, USA) at room temperature for $1 \mathrm{~h}$. The stained tissues slides were then observed under a light microscope. The expression levels of PKM2 were evaluated using an IHC score by multiplying the frequency and intensity scores. The frequency score for PKM2 expression was graded according to the percentage of positively stained tumor cells $(<10 \%=0,10-25 \%=1,26-50 \%=2$, and $>50 \%=3)$. The intensity of PKM2 expression was scored as negative $=0$, weak $=1$, moderate $=2$ and strong=3. The IHC scores for PKM2 expression were then categorized as high (IHC score $\geq 6$ ) or low (IHC score $<6$ ).

Western blotting. A total of $20 \mu \mathrm{g}$ protein from CCA cell lines were loaded into $10 \%$ sodium dodecyl sulphate-polyacrylamide gel electrophoresis media and transferred onto Hybond ${ }^{\mathrm{TM}}-\mathrm{P}$ membranes (GE Healthcare, Buckinghamshire, UK). The membranes were probed with primary antibody to PKM2 $(1: 8,000)$ at room temperature for $1 \mathrm{~h}$ and then secondary antibody (GE Healthcare) for $1 \mathrm{~h}$ at room temperature. The PKM2 expression signals were detected using an enhanced chemiluminescence prime Western blotting detection kit (GE Healthcare). Image analysis was performed using Image Quant ${ }^{\mathrm{TM}}$ Imager (GE Healthcare). Relative band intensities were quantified and normalized to those for $\beta$-actin.

Cell proliferation and clonogenic assay. Briefly, $2 \times 10^{3}$ cells/well were seeded in triplicate into 96-well plates and incubated overnight. Cells were then treated with $0.5,1$ and $1.5 \mu \mathrm{M}$ PKM2 inhibitor shikonin (Sigma-Aldrich, St. Louis, MO, USA). After treatment, 5 $\mathrm{mg} / \mathrm{ml}$ MTT (3-(4,5-dimethylthiazol-2-yl)-2,5-diphenyltetrazolium bromide) (Invitrogen Life Technologies, Carlsbad, CA, USA) was added and incubated at $37^{\circ} \mathrm{C}$ for $4 \mathrm{~h}$. The formazan crystals were dissolved with dimethyl sulfoxide. The absorbance was monitored with a microplate reader (SUNRISE, Groedig, Austria) at a wavelength of $540 \mathrm{~nm}$. The half-maximal inhibitory concentration $\left(\mathrm{IC}_{50}\right)$ of cells treated with shikonin for 24,48 and $72 \mathrm{~h}$ for each cell line was also analyzed. In order to test whether the effect of shikonin on growth was associated with ROS production, the antioxidant $\mathrm{N}$ acetyl cysteine (NAC) was used in this study. The viability of CCA cells was examined using the sulforhodamine B assay (SigmaAldrich) as previously described (23). In addition, cell proliferation was also determined by a colony-formation assay. CCA cells were seeded at $1.5 \times 10^{4}$ cells into 6 -well plates and cultured in an incubator 
with $5 \% \mathrm{CO}_{2}$ at $37^{\circ} \mathrm{C}$, overnight. After being treated with 0.5 , 1 , or $1.5 \mu \mathrm{M}$ shikonin for $24 \mathrm{~h}$, a total of 80 cells/well were seeded into 12 -well plates and incubated at $5 \% \mathrm{CO}_{2}$ at $37^{\circ} \mathrm{C}$ for 7 days with the media changed every 3 days. Colonies were then fixed with $4 \%$ paraformaldehyde for $30 \mathrm{~min}$ at room temperature, and stained with $0.5 \%$ crystal violet for $30 \mathrm{~min}$. The number of colonies were then counted under microscopy.

Trypan blue dye exclusion assay. Briefly, $5 \times 10^{3}$ cells were plated into 24-well plates, followed by treatment with 0.5 and $1 \mu \mathrm{M}$ shikonin for $48 \mathrm{~h}$. The cells were trypsinized and the ratios of dead and viable cells were determined by trypan blue staining by hemocytometer.

Migration assay. In brief, $4 \times 10^{4}$ cells were pre-treated with shikonin at $0.25,0.5 \mu \mathrm{M}$ or without shikonin for $24 \mathrm{~h}$ in serum-free medium were plated onto the upper chamber of Transwell plates. The lower chambers contained complete medium used as a chemo-attractant. The migrated cells were fixed with $4 \%$ paraformaldehyde, and stained with $0.4 \%$ sulforhodamine B. The number of migrated cells were photographed and counted using KKU Cancer Count Software (24). The experiments were performed in triplicate using three independent-experiments.

Measurement of ROS production. Intracellular ROS levels were measured using flow cytometry with chloromethyl 2', 7'dichlorodihydrofluorescein diacetate (CM-H2DCFDA) (Invitrogen Life Technologies). In brief, $1 \times 10^{5}$ cells were seeded into 12 -well plates and incubated at $37^{\circ} \mathrm{C}$ with $5 \% \mathrm{CO}_{2}$ for $24 \mathrm{~h}$. The culture medium was removed and cells were treated with $1 \mu \mathrm{M}$ of shikonin, 5 or $10 \mathrm{mM} \mathrm{NAC}$, or a combination of shikonin with NAC for $2 \mathrm{~h}$. Cells were then incubated with $2.5 \mu \mathrm{M}$ CM-H2DCFDA for $30 \mathrm{~min}$ in serum-free media. At the end of the incubation period, cells were trypsinized, followed by centrifugation $2400 \times g$ at $4^{\circ} \mathrm{C}$ for $5 \mathrm{~min}$. The supernatant was discarded and the cell pellet resuspended with 1X PBS was transferred to Falcon tubes after being filtered through a nylon mesh. The fluorescence intensity was assessed using a BD FACSCanto $^{\mathrm{TM}}$ II flow cytometer (BD Biosciences, Franklin Lakes, NJ, USA). The data was analyzed by FlowJo program (BD Biosciences) and presented as the mean fluorescent intensity $(\mathrm{MFI}) \pm \mathrm{SD}$ of three replicates.

Statistical analysis. The mean values \pm standard deviation (SD) from three independent experiments are presented. Data were compared using two-tailed Student's $t$-tests to compare the differences between two groups. Survival analysis was performed by KaplanMeier method with log-rank test. The association between PKM2 expression in CCA tissues and clinicopathological findings were analyzed using Pearson's chi-square test. A $p$-value less than 0.05 was considered as indicating statistical significance.

\section{Results}

PKM2 was overexpressed in CCA tissues. To further determine the role of PKM2 and CCA carcinogenesis, the expression of PKM2 was investigated by IHC. It was found that PKM2 expression was gradually up-regulated during cholangiocarcinogenesis as demonstrated in the liver flukeassociated hamster model of CCA. The expression of PKM2 was predominantly localized in the cytosol with less in the nuclear counterpart. PKM2 was weakly expressed in normal bile ducts of control and NDMA-treated groups during all periods of experimentation. PKM2 was slightly expressed in the hyperplastic bile duct of the OV-treated group, at 1 month and highly expressed at 3 and 6 months. In contrast, PKM2 was highly expressed in tumoral area that were found in the group treated with OV plus NDMA starting at 1 month of experimentation (Figure 1). The IHC indices of PKM2 expression in hamster tissues are shown in Figure 1. The percentage of PKM2-positive staining and the histological classification including normal bile duct, hyperplastic bile duct and CCA in OV-associated CCA of hamster tissues are summarized in Table I. PKM2 was weakly expressed in almost all hyperplastic bile ducts of each group, while it was highly expressed in 75\% (29/41) of cancer areas of the group treated with OV plus NDMA. PKM2 expression levels in bile duct hyperplasia and cancer were significantly higher compared to the adjacent normal bile ducts. Moreover, the expression of PKM2 was further confirmed in CCA tissues from patients $(n=80)$. Stronger PKM2 expression was found in CCA ducts compared to the adjacent normal bile ducts. PKM2-positive ducts were found in 25\% (19/76), $43 \%$ $(34 / 80)$, and $93 \%(74 / 80)$ in areas of normal, hyperplastic and CCA tissue. CCA patient tissues were divided into two groups with high PKM2 expression (IHC score $\geq 6$ ) and low PKM2 expression (IHC score $<6$ ). The association between PKM2 expression levels and clinicopathological findings were determined. A high expression of PKM2 was detected in $61 \%(45 / 74)$ of CCA tissues. Conversely, PKM2 expression was low in $30 \%(6 / 20)$ of normal bile duct epithelial cells. The expression of PKM2 was frequently localized cytoplasm (77/80, 96\%), whereas nuclear expression was detected in only 3/80 (4\%) samples. This finding suggests that expression of PKM2 is associated with the carcinogenic processes of CCA. Representative IHC staining of PKM2 is shown in Figure 2.

The correlations between PKM2 expression and clinicopathological features were analyzed using the chisquare test and survival analysis. Based on the IHC score, high PKM2 expression was significantly associated with metastasis $(p<0.05)$ (Table II). No association between PKM2 expression and overall survival of patients was observed (data not shown).

Shikonin significantly suppressed cell proliferation and induced death of CCA cells. Firstly, the expression of PKM2 protein in three CCA cell lines of differing was quantified using western blotting analysis. PKM2 was more highly expressed in KKU-100 and KKU-213A cells, while it was less expressed in KKU-055 cells (Figure 3A). Secondly, antitumor activity of shikonin was determined in three CCA cell lines. Shikonin suppressed CCA cell viability in a doseand time-dependent manner compared to vehicle-treated cells 


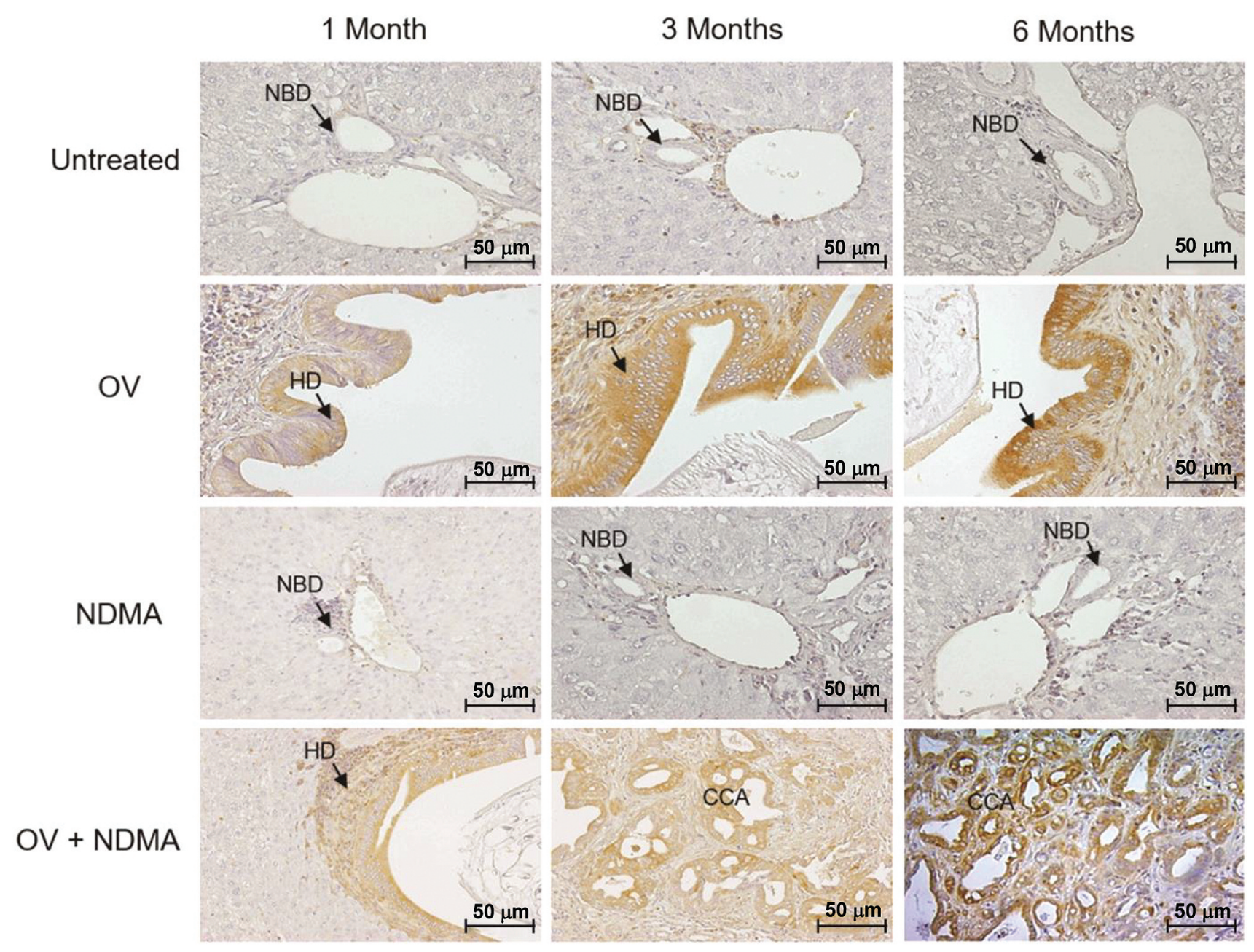

Figure 1. Pyruvate kinase M2 (PKM2) expression in tissue from the liver fluke (Opisthorchis viverrini; OV)-associated cholangiocarcinoma (CCA) hamster model using immunohistochemistry. PKM2 expression increased with steps of cholangiocarcinoma (CCA) carcinogenesis. In the untreated group, very low PKM2 expression was observed in normal bile duct (NBD). In the N-nitrosodimethylamine (NDMA)-treated group, PKM2 was slightly expressed in NBD. In the OV-infected group, high PKM2 expression was observed in hyperplastic bile duct (HD). In contrast, in the group treated with OV plus NDMA, the highest PKM2 expression was determined in tumoral areas at 3 and 6 months.

(Figure 3B). The $\mathrm{IC}_{50}$ values for shikonin against these three cell lines are provided in Table III. KKU-213A cell line exhibited more resistance to shikonin than the other two CCA cell lines. The colony-formation assay, which represents the ability for colony formation and proliferation, revealed that all CCA cell lines treated with shikonin produced significantly fewer colonies than the vehicle treated cells (Figure 3C). The effects of shikonin on cell death were further assessed. CCA cells were cultured with and without shikonin for $48 \mathrm{~h}$. Shikonin treatment of CCA cells led to a significantly higher number of cell deaths than in control cells, in a dose-dependent manner $(p<0.05)$ (Figure 3D).

Shikonin markedly reduced the migration ability of CCA cells. The role of shikonin on cell motility was determined using a Boyden chamber-based cell migration assay. CCA cells were treated with 0.25 and $0.5 \mu \mathrm{M}$ shikonin for $24 \mathrm{~h}$ and the numbers of migrated cells were counted. The migration of KKU-100 and KKU-055 cell lines treated with $0.5 \mu \mathrm{M}$ shikonin were significantly reduced more than 2 -fold when compared to the controls $(p<0.05)$. The significant anti-migration effect of shikonin was observed in all three CCA cell lines (Figure 3E).

Shikonin inhibited CCA cell proliferation via increased ROS production. Recently, many studies have reported that shikonin inhibits proliferation (25), anti-apoptosis (25-27), and migration (28) of cancer cells via the induction of ROS. Therefore, an investigation as to whether shikonin-inhibited 

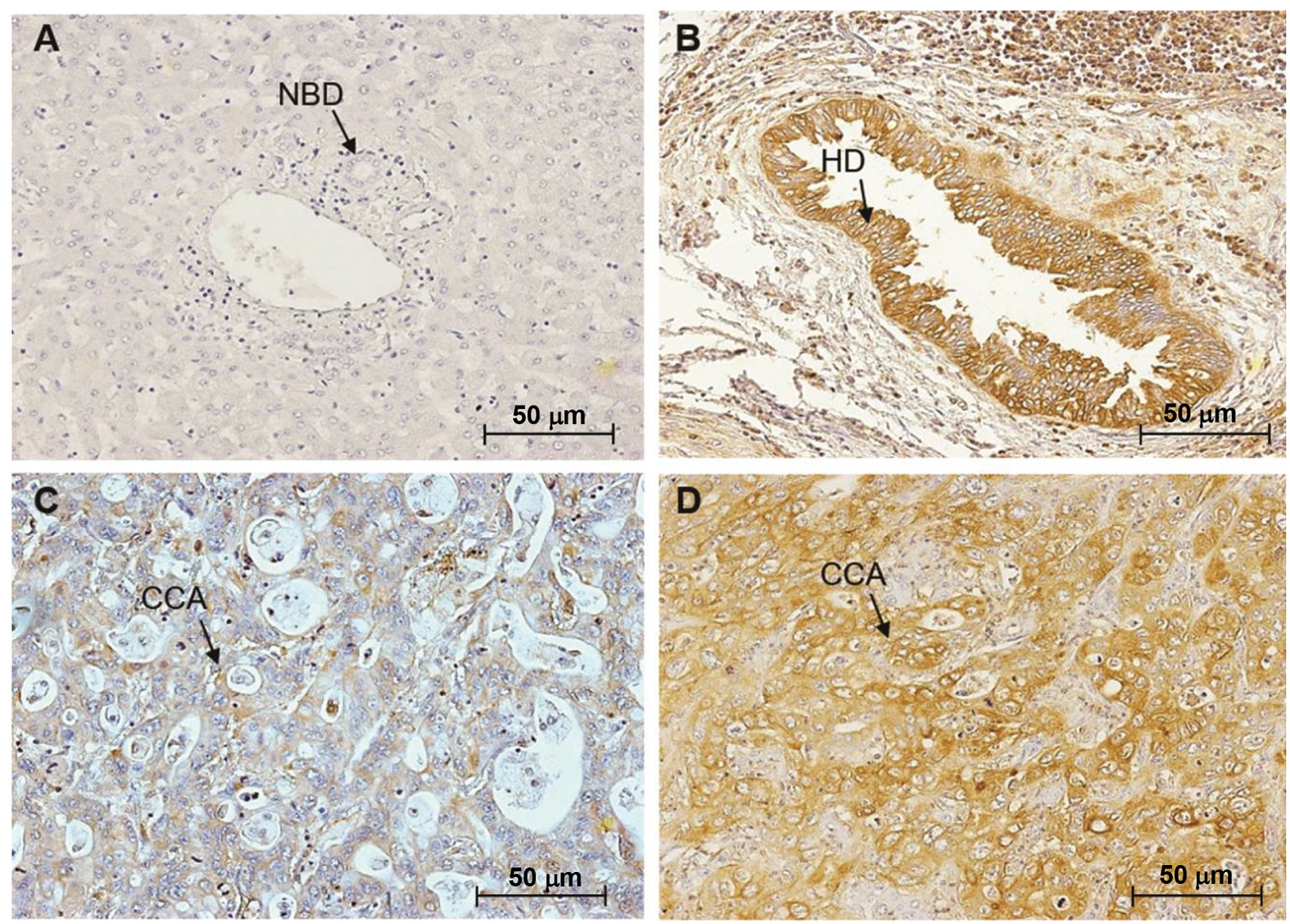

Figure 2. Pyruvate kinase M2 (PKM2) expression in tissues from patients with cholangiocarcinoma (CCA) as revealed by immunohistochemistry $($ magnification $\times 200)$ in normal bile duct $(N B D)(A)$, hyperplastic bile duct $(H D)(B)$ and CCA tumoral area $(C$ and $D)$.

Table I. The histological classification of pyruvate kinase M2 (PKM2) expression in the liver fluke (Opisthorchis viverrini)-associated cholangiocarcinoma (CCA) hamster tissues.

\begin{tabular}{lccccc}
\hline & \multicolumn{4}{c}{ Treatment group, $\mathrm{n}$} \\
\cline { 2 - 4 } Histology & Untreated & OV & NDMA & OV + NDMA & Total positive (OV + NDMA) \\
\hline NBD & 20 & 20 & 20 & 60 & $40 \%(24 / 60)$ \\
PKM2-positive & 10 & 10 & 4 & 24 & $71 \%(29 / 41)$ \\
HD & 12 & 14 & 15 & 41 & $75 \%(6 / 8)$ \\
PKM2-positive & 10 & 10 & 9 & 8 & 7 \\
CCA & - & 3 & 5 & 6 & 70 \\
PKM2-positive & - & 2 & 4 & & 70 \\
\hline
\end{tabular}

NBD: Normal bile duct, NDMA: $N$-Nitrosodimethylamine-treated group, OV: Opisthorchis viverrini-infected group, HD: hyperplastic bile duct.

CCA cell proliferation and migration might be attributed to the production of ROS was carried out using NAC as an ROS scavenger in this experiment. Based on their high PKM2 protein expression, KKU-100 and KKU-213A cell lines were used for further studies. The antiproliferative activity of shikonin was significantly reduced when applied in combination with NAC when compared to that with shikonin treatment alone (Figure 4A). This result reveals that 
Table II. Pyruvate kinase M2 (PKM2) expression and clinicopathological findings of patients with cholangiocarcinoma (CCA).

\begin{tabular}{|c|c|c|c|c|}
\hline \multirow[t]{2}{*}{ Clinical characteristic } & \multirow[b]{2}{*}{$\begin{array}{l}\text { No. of } \\
\text { patients }\end{array}$} & \multicolumn{2}{|c|}{$\begin{array}{c}\text { PKM2 expression, } \\
\mathrm{n}(\%)\end{array}$} & \multirow[b]{2}{*}{$p$-Value } \\
\hline & & Low & High & \\
\hline \multicolumn{5}{|l|}{ Age $(n=80)$} \\
\hline$<56$ Years & 38 & $16(42.1)$ & $22(57.9)$ & \multirow[t]{2}{*}{0.658} \\
\hline$\geq 56$ Years & 42 & $20(47.6)$ & $22(52.4)$ & \\
\hline \multicolumn{5}{|l|}{ Gender $(n=80)$} \\
\hline Male & 54 & $24(44.4)$ & $30(55.6)$ & \multirow[t]{2}{*}{$>0.999$} \\
\hline Female & 26 & $12(46.2)$ & $14(53.8)$ & \\
\hline \multicolumn{5}{|l|}{ Histological type $(n=80)$} \\
\hline Non-papillary & 53 & $21(39.6)$ & $32(60.4)$ & \multirow[t]{2}{*}{0.236} \\
\hline Papillary & 27 & $15(55.6)$ & $12(44.4)$ & \\
\hline \multicolumn{5}{|l|}{ T-Stage $(n=80)$} \\
\hline $\mathrm{T} 1$ & 6 & $3(50.0)$ & $3(50.0)$ & \multirow[t]{4}{*}{0.925} \\
\hline $\mathrm{T} 2$ & 9 & $4(44.4)$ & $5(55.6)$ & \\
\hline $\mathrm{T} 3$ & 33 & $16(48.5)$ & $17(51.5)$ & \\
\hline $\mathrm{T} 4$ & 32 & $13(40.6)$ & $19(59.4)$ & \\
\hline \multicolumn{5}{|l|}{$N$-Stage $(n=69)$} \\
\hline No & 36 & $21(58.3)$ & 15 (41.7) & \multirow[t]{2}{*}{0.150} \\
\hline N1 & 33 & $13(39.4)$ & $20(60.6)$ & \\
\hline \multicolumn{5}{|l|}{ M-Stage $(n=73)$} \\
\hline M0 & 64 & $32(50.0)$ & $32(50.0)$ & \multirow[t]{2}{*}{0.035} \\
\hline M1 & 9 & $1(11.1)$ & 8 (88.9) & \\
\hline \multicolumn{5}{|l|}{ Tumor stage $(n=80)$} \\
\hline $\mathrm{I}+\mathrm{II}$ & 11 & $5(45.5)$ & $6(54.5)$ & \multirow[t]{2}{*}{$>0.999$} \\
\hline III + IV & 69 & $31(44.9)$ & $38(55.1)$ & \\
\hline \multicolumn{5}{|l|}{ Tumor size $(\mathrm{n}=80)$} \\
\hline$<7 \mathrm{~cm}$ & 38 & $14(36.8)$ & $24(63.2)$ & \multirow[t]{2}{*}{0.184} \\
\hline$\geq 7 \mathrm{~cm}$ & 42 & $22(52.9)$ & $20(47.6)$ & \\
\hline
\end{tabular}

The association between PKM2 expression and clinicopathological findings were analyzed using chi-squared test. Statistically significant $p$-values are shown in bold.

the antiproliferative effect of shikonin on CCA cell lines was mediated by an increase of ROS production. To further confirm this effect resulted from an alteration of ROS production, intracellular ROS levels were measured with CM-H2DCFDA stain followed by flow cytometry. The results indicated that ROS production was significantly increased in CCA cells treated with $1 \mu \mathrm{M}$ shikonin for $2 \mathrm{~h}$ compared with the control cells, whereas, ROS levels were significantly reduced to nearly basal levels in cells treated with shikonin in combination with NAC compared to both treatments alone (Figure 4B). These findings suggest that shikonin-induced ROS production is essential for inhibition of CCA cell proliferation.

\section{Discussion}

The most effective therapeutic approach for CCA is curative resection. This strategy, however, remains limited, since most
Table III. Half-maximal inhibitory concentration $\left(I C_{50}\right)$ values for shikonin treatment against cell lines. Date are the mean \pm standard deviation.

\begin{tabular}{lccc}
\hline Cell line & \multicolumn{3}{c}{$\mathrm{IC}_{50}(\mu \mathrm{M})$} \\
\cline { 2 - 4 } & $24 \mathrm{~h}$ & $48 \mathrm{~h}$ & $72 \mathrm{~h}$ \\
\hline KKU-100 & $0.84 \pm 0.02$ & $0.61 \pm 0.03$ & $0.53 \pm 0.00$ \\
KKU-055 & $0.82 \pm 0.02$ & $0.59 \pm 0.04$ & $0.53 \pm 0.05$ \\
KKU-213A & $0.91 \pm 0.10$ & $0.77 \pm 0.13$ & $0.51 \pm 0.02$ \\
\hline
\end{tabular}

patients with CCA are often diagnosed with disease that is typically unresectable. The standard treatment for these patients is chemotherapy/radiotherapy. Resistance to prolonged use of conventional chemotherapeutic agents and disease recurrence are a major problem in CCA that contribute to poor outcomes of patients $(4,29)$. Therefore, development of new therapeutic targets and effective drugs for CCA are needed.

In the present study, it was demonstrated that overexpression of PKM2 is associated with CCA development in liver fluke-associated CCA. Increased PKM2 expression has been reported in a wide range of tumors and inflammatory disorders. OV infection can cause chronic inflammation and oxidative stress by both mechanical and immune responses, leading to cellular/tissue damage. For instance, increased immune cell activity, and production of IL1 $\beta$ and IL6 proinflammatory cytokines, and growth factor occur in response to $\mathrm{OV}$ infection. PKM2 is an enzyme that plays an important role in glycolysis and is predominantly expressed during embryonic development, in highly proliferating cells, and in cancer cells $(30,31)$. It was recently reported that increased inflammatory signaling and PKM2 expression contribute to tumorigenesis of hilar cholangiocarcinoma induced by chronic inflammation (32). Additionally, high PKM2 expression was significantly associated with the M1 stage in patients with CCA. This clinical observation agrees with that in other cancer types (10, 12, 32). Over the past decade, the evidence has indicated that genetic alteration by activation of oncogenes, and inactivation of tumor-suppressor genes may regulate expression and functions of PKM2. HIF1 $\alpha$ is a transcription regulator that predominantly induces transcription of genes targeted by HIF1 $\alpha$, including PKM2, which contributes to increased PKM2 expression levels in cancer cells (33). Therefore, these findings may support the existence of a functional role of PKM2 in development and progression of CCA and indicate that PKM2 may be a potential therapeutic target in CCA.

It has been shown that shikonin and its derivatives can inhibit cell proliferation, migration, and invasion and induce apoptosis in various cancer types $(34,35)$. Moreover, a 

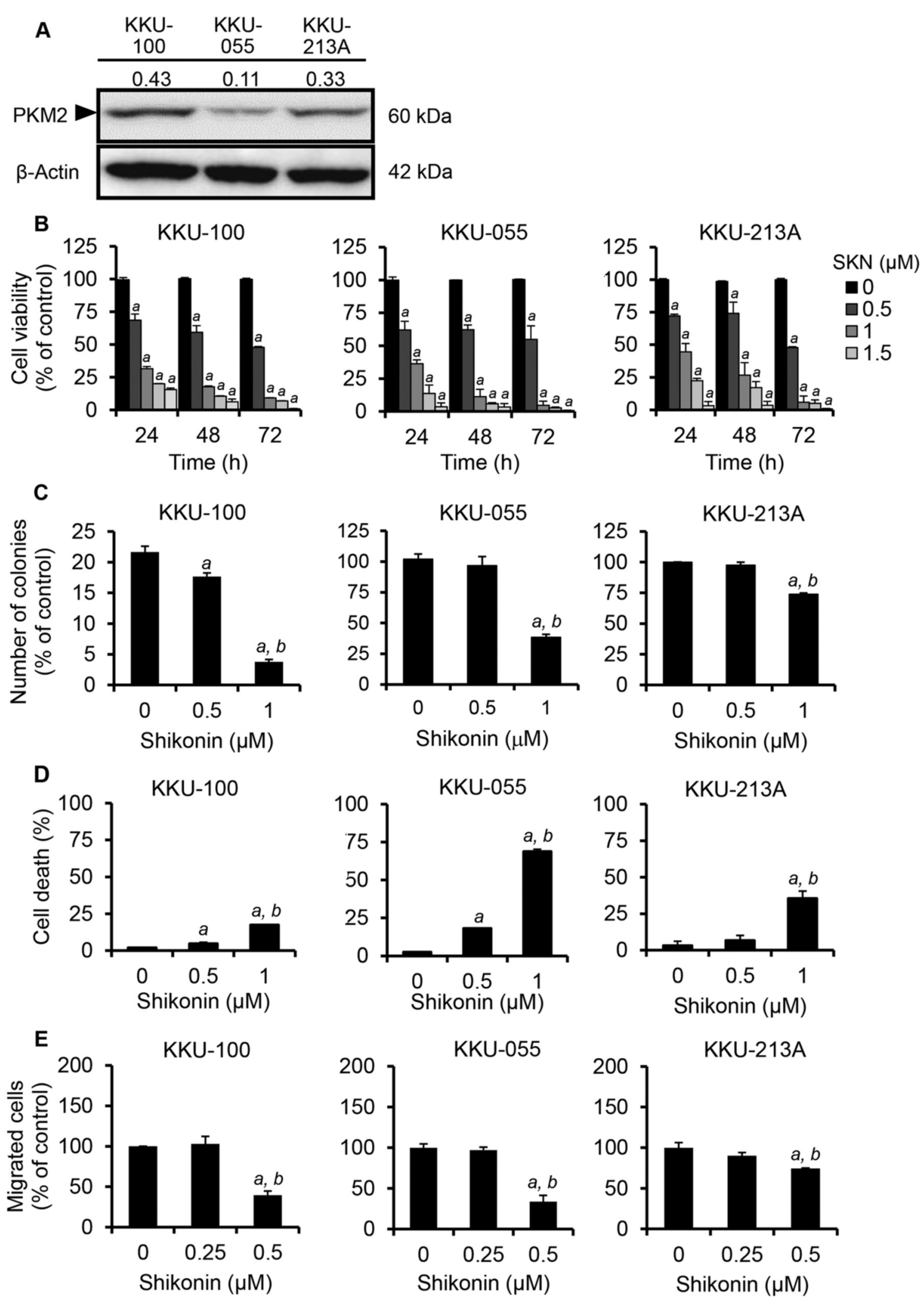

Figure 3. Antitumor activity of shikonin on cholangiocarcinoma (CCA) cell lines. PKM2 expression levels in CCA cells using western blot analysis $(A)$. Shikonin inhibited cell proliferation $(B)$, colony formation $(C)$, migration $(E)$ and increased death of CCA cells $(D)$. Significantly different at p<0.05 vs. ${ }^{a}$ control, ${ }^{b} 0.5 \mu M$ shikonin. 
A
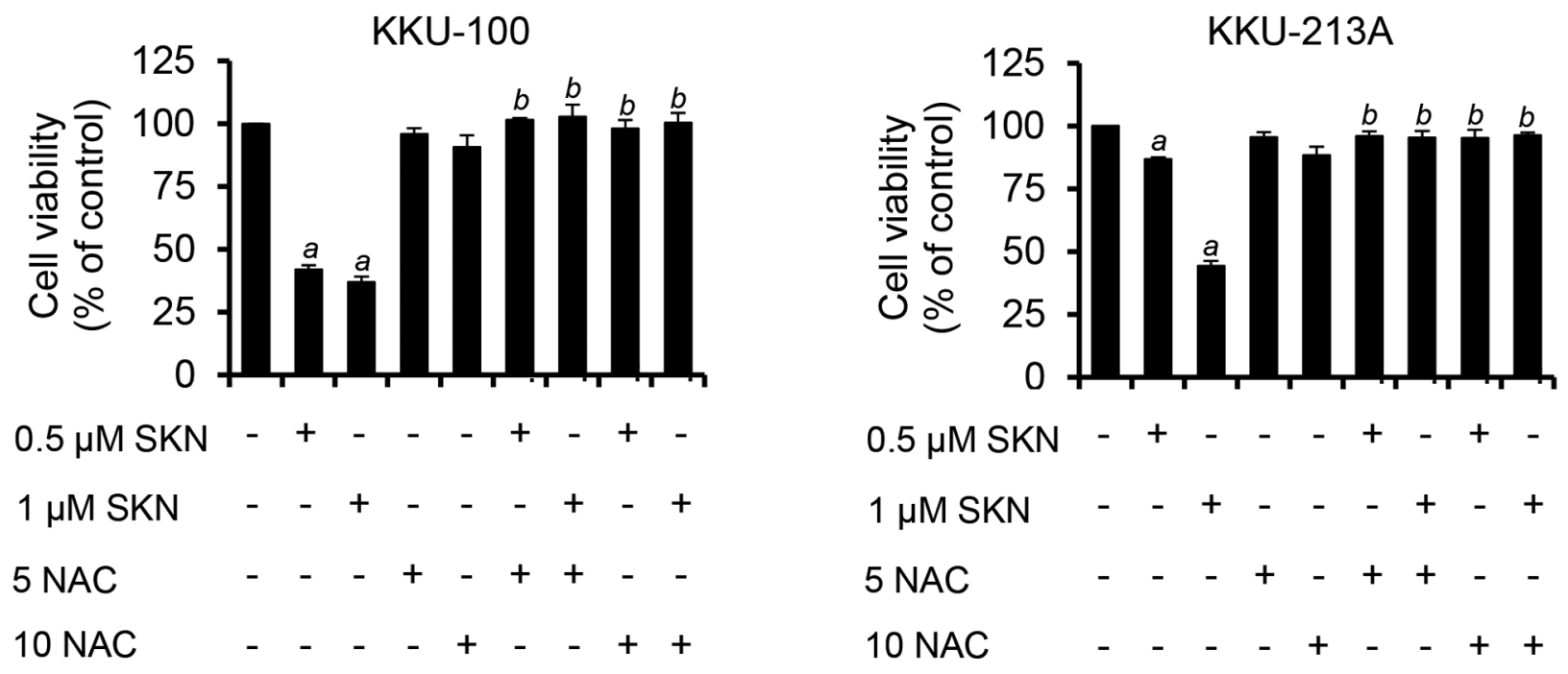

B

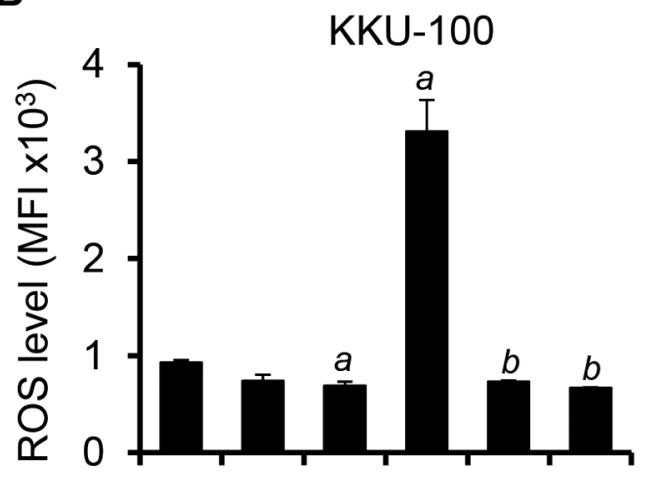

$1 \mu \mathrm{M}$ SKN $-\quad-\quad+\quad+$

$5 \mathrm{NAC}-+-++-$

10 NAC - $\quad-\quad+\quad-\quad+$

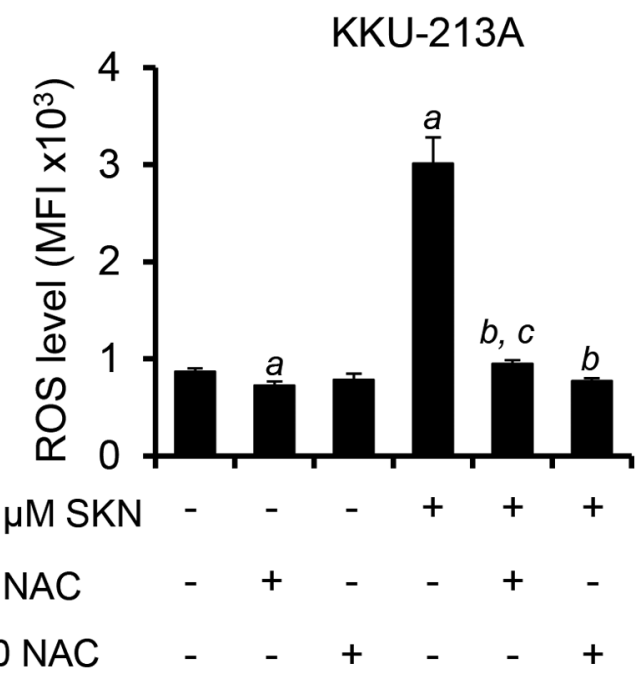

Figure 4. Shikonin (SKN) inhibited cholangiocarcinoma (CCA) cell proliferation via reactive oxygen species (ROS) production. Proliferation assay in CCA cell lines treated with SKN and/or N-acetylcysteine (NAC) (A). SKN significantly inhibited growth of CCA cell lines. However, the CCA cell proliferation was dramatically increased in SKN combination with NAC. SKN treatment significantly induced ROS production compared to the untreated control, whereas using SKN plus NAC, the ROS level was significantly reduced in both CCA cell lines. MFI: Mean fluorescence intensity. Significantly different at $p<0.05 v s .{ }^{a}$ control, ${ }^{b} S K N$ alone, ${ }^{c} N A C$ alone.

clinical trial revealed shikonin to be an effective treatment in patients with later-stage lung cancer (36). Herein, the antitumor effect and the underlying molecular mechanisms of shikonin in CCA were investigated. It was found that shikonin inhibited CCA cell proliferation and migration while inducing death in a dose-dependent manner via ROS induction. Inhibition of CCA cell proliferation and migration by shikonin were rescued by NAC treatment. These results are consistent with previous reports showing that shikonin inhibited cell growth and induced apoptosis of CCA cells via increased ROS $(37,38)$. ROS plays an important role in various diseases, including cancer mediated by different mechanisms and signaling pathways involved in cell proliferation, migration, apoptosis, and senescence. In cancer suppression, shikonin induced ROS to cytotoxic levels, resulting in inhibition of cell proliferation, migration, invasion and chemoresistance, whereas it induced cell senescence and apoptosis in several cancer types (37-39). 
Lui et al. reported that inhibition of ROS with NAC attenuated shikonin-induced cell growth inhibition by inducing $\mathrm{G}_{2}$ /M-phase arrest and reducing endoplasmic reticulum stress and phosphorylation of p38 (25).

In summary, the key findings in the present study revealed that overexpression of PKM2 is required for tumorigenesis, proliferation, and migration of CCA cells. Moreover, shikonin can inhibit proliferation, migration and induce death of CCA cells via ROS overproduction.

\section{Conclusion}

Inhibition of PKM2 by shikonin may useful to control progressive phenotypes of CCA. Shikonin might be a promising anticancer agent for treatment of CCA by acting as a potent inducer of ROS.

\section{Conflicts of Interest}

The Authors declare they have no conflicts of interest in regard to this study.

\section{Authors' Contributions}

UT, WS, SW and CW designed the study. WS, SW and CW supervised the study. UT, TB and UC performed the assays. UT, WS, SaW and SW analyzed the experimental data. UT, WS, SW and $\mathrm{CW}$ wrote the article.

\section{Acknowledgements}

The Authors acknowledge Senior Research Scholar Grant, Thailand Research Fund/KKU (RTA5780012), Khon Kaen University (KKU580604), Faculty of Medicine (IN58208), Cholangiocarcinoma Research Institute (CARI), and the Research Instrument Center, Khon Kaen University, for the service support of the flow cytometer. We would like to acknowledge Professor James Arthur Will for editing the draft article via Publication Clinic KKU, Thailand.

\section{References}

1 Khan SA, Tavolari S and Brandi G: Cholangiocarcinoma: Epidemiology and risk factors. Liver Int 39(Suppl 1): 19-31, 2019. PMID: 30851228. DOI: 10.1111/liv.14095

2 Thamavit W, Bhamarapravati N, Sahaphong S, Vajrasthira S and Angsubhakorn S: Effects of dimethylnitrosamine on induction of cholangiocarcinoma in opisthorchis viverrini-infected syrian golden hamsters. Cancer Res 38(12): 4634-4639, 1978. PMID: 214229.

3 Sriputtha S, Khuntikeo N, Promthet S and Kamsa-Ard S: Survival rate of intrahepatic cholangiocarcinoma patients after surgical treatment in thailand. Asian Pac J Cancer Prev 14(2): 1107-1110, 2013. PMID: 23621195. DOI: 10.7314/apjcp.2013.14.2.1107

4 Thongprasert S: The role of chemotherapy in cholangiocarcinoma. Ann Oncol 16 Suppl 2: ii93-96, 2005. PMID: 15958484. DOI: 10.1093/annonc/mdi712
5 Hanahan D and Weinberg RA: Hallmarks of cancer: The next generation. Cell 144(5): 646-674, 2011. PMID: 21376230. DOI: 10.1016/j.cell.2011.02.013

6 Madhok BM, Yeluri S, Perry SL, Hughes TA and Jayne DG: Targeting glucose metabolism: An emerging concept for anticancer therapy. Am J Clin Oncol 34(6): 628-635, 2011. PMID: 20805739. DOI: 10.1097/COC.0b013e3181e84dec

7 Mazurek S: Pyruvate kinase type m2: A key regulator of the metabolic budget system in tumor cells. Int J Biochem Cell Biol 43(7): 969-980, 2011. PMID: 20156581. DOI: 10.1016/ j.biocel.2010.02.005

8 Christofk HR, Vander Heiden MG, Harris MH, Ramanathan A, Gerszten RE, Wei R, Fleming MD, Schreiber SL and Cantley LC: The $\mathrm{m} 2$ splice isoform of pyruvate kinase is important for cancer metabolism and tumour growth. Nature 452(7184): 230233, 2008. PMID: 18337823. DOI: 10.1038 /nature06734

9 Hsu MC and Hung WC: Pyruvate kinase m2 fuels multiple aspects of cancer cells: From cellular metabolism, transcriptional regulation to extracellular signaling. Mol Cancer 17(1): 35, 2018. PMID: 29455645. DOI: 10.1186/s12943-018-0791-3

10 Cui R and Shi XY: Expression of pyruvate kinase $\mathrm{m} 2$ in human colorectal cancer and its prognostic value. Int J Clin Exp Pathol 8(9): 11393-11399, 2015. PMID: 26617865. DOI: 19362625/IJCEP0013130

11 Mohammad GH, Olde Damink SW, Malago M, Dhar DK and Pereira SP: Pyruvate kinase $\mathrm{m} 2$ and lactate dehydrogenase a are overexpressed in pancreatic cancer and correlate with poor outcome. PLoS One 11(3): e0151635, 2016. PMID: 26989901. DOI: $10.1371 /$ journal.pone.0151635

12 Kobierzycki C, Piotrowska A, Latkowski K, Zabel M, NowakMarkwitz E, Spaczynski M, Kedzia W, Pula B, PodhorskaOkolow M and Dziegiel P: Correlation of pyruvate kinase $\mathrm{m} 2$ expression with clinicopathological data in ovarian cancer. Anticancer Res 38(1): 295-300, 2018. PMID: 29277786. DOI: 10.21873/anticanres.12221

13 Miao Y, Lu M, Yan Q, Li S and Feng Y: Inhibition of proliferation, migration, and invasion by knockdown of pyruvate kinase-m2 $(\mathrm{pkm} 2)$ in ovarian cancer skov3 and ovcar3 cells. Oncol Res 24(6): 463-475, 2016. PMID: 28281967. DOI: $10.3727 / 096504016 \mathrm{X} 14685034103671$

14 Wang Y, Hao F, Nan Y, Qu L, Na W, Jia C and Chen X: Pkm2 inhibitor shikonin overcomes the cisplatin resistance in bladder cancer by inducing necroptosis. Int J Biol Sci 14(13): 18831891, 2018. PMID: 30443191. DOI: 10.7150/ijbs.27854

15 Granchi C and Minutolo F: Anticancer agents that counteract tumor glycolysis. ChemMedChem 7(8): 1318-1350, 2012. PMID: 22684868. DOI: $10.1002 / \mathrm{cmdc} .201200176$

16 Chen X, Yang L, Oppenheim JJ and Howard MZ: Cellular pharmacology studies of shikonin derivatives. Phytother Res 16(3): 199-209, 2002. PMID: 12164262. DOI: 10.1002/ptr.1100

17 Liu C, Yin L, Chen J and Chen J: The apoptotic effect of shikonin on human papillary thyroid carcinoma cells through mitochondrial pathway. Tumour Biol 35(3): 1791-1798, 2014. PMID: 24081676. DOI: 10.1007/s13277-013-1238-5

18 Liu JP, Liu D, Gu JF, Zhu MM and Cui L: Shikonin inhibits the cell viability, adhesion, invasion and migration of the human gastric cancer cell line mgc-803 via the toll-like receptor 2/nuclear factor-kappa b pathway. J Pharm Pharmacol 67(8): 1143-1155, 2015. PMID: 25880237. DOI: 10.1111/ jphp.12402 
19 Sripa B, Leungwattanawanit S, Nitta T, Wongkham C, Bhudhisawasdi V, Puapairoj A, Sripa C and Miwa M: Establishment and characterization of an opisthorchiasisassociated cholangiocarcinoma cell line (kku-100). World J Gastroenterol 11(22): 3392-3397, 2005. PMID: 15948244. DOI: 10.3748/wjg.v11.i22.3392

20 Sripa B, Seubwai W, Vaeteewoottacharn K, Sawanyawisuth K, Silsirivanit A, Kaewkong W, Muisuk K, Dana P, Phoomak C, LertItthiporn W, Luvira V, Pairojkul C, Teh BT, Wongkham S, Okada $\mathrm{S}$ and Chamgramol Y: Functional and genetic characterization of three cell lines derived from a single tumor of an opisthorchis viverrini-associated cholangiocarcinoma patient. Hum Cell, 2020. PMID: 32207095. DOI: 10.1007/s13577-020-00334-w

21 Boonmars T, Boonjaraspinyo S and Kaewsamut B: Animal models for opisthorchis viverrini infection. Parasitol Res 104(3): 701-703, 2009. PMID: 19050927. DOI: 10.1007/s00436-0081268-x

22 Thonsri U, Seubwai W, Waraasawapati S, Sawanyawisuth K, Vaeteewoottacharn $\mathrm{K}$, Boonmars $\mathrm{T}$ and Cha'on $\mathrm{U}$ : Overexpression of lactate dehydrogenase a in cholangiocarcinoma is correlated with poor prognosis. Histol Histopathol 32(5): 503-510, 2017. PMID: 27615379. DOI: 10.14670/HH-11819

23 Wongkham S, Junking M, Wongkham C, Sripa B, Chur-In S and Araki N: Suppression of galectin-3 expression enhances apoptosis and chemosensitivity in liver fluke-associated cholangiocarcinoma. Cancer Sci 100(11): 2077-2084, 2009. PMID: 19723119. DOI: 10.1111/j.1349-7006.2009.01304.x

24 Namwong Korwit, Polpinit Pattarawit, Wongkham Sopit and Charupong S: Modified watershed transform algorithm for cancer cell segmentation counting. KKU Eng J 43(S2): 370-372, 2016. DOI: 10.14456/kkuenj.2016.132

25 Liu T, Sun X and Cao Z: Shikonin-induced necroptosis in nasopharyngeal carcinoma cells via ros overproduction and upregulation of ripk $1 / \mathrm{ripk} 3 / \mathrm{mlkl}$ expression. Onco Targets Ther 12 : 2605-2614, 2019. PMID: 31118661. DOI: 10.2147/OTT.S200740

26 Huang WR, Zhang Y and Tang X: Shikonin inhibits the proliferation of human lens epithelial cells by inducing apoptosis through ros and caspase-dependent pathway. Molecules 19(6): 7785-7797, 2014. PMID: 24962386. DOI: 10.3390/molecules 19067785

27 Zhou G, Yang Z, Wang X, Tao R and Zhou Y: Trail enhances shikonin induced apoptosis through ros/jnk signaling in cholangiocarcinoma cells. Cell Physiol Biochem 42(3): 10731086, 2017. PMID: 28662515. DOI: $10.1159 / 000478758$

28 Chen Y, Zheng L, Liu J, Zhou Z, Cao X, Lv X and Chen F: Shikonin inhibits prostate cancer cells metastasis by reducing matrix metalloproteinase-2/-9 expression via akt/mtor and ros/erk 1/2 pathways. Int Immunopharmacol 21(2): 447-455, 2014. PMID: 24905636. DOI: 10.1016/j.intimp.2014.05.026

29 Ramirez-Merino N, Aix SP and Cortes-Funes H: Chemotherapy for cholangiocarcinoma: An update. World J Gastrointest Oncol 5(7): 171-176, 2013. PMID: 23919111. DOI: 10.4251/wjgo.v5.i7.171
30 Sripa B, Thinkhamrop B, Mairiang E, Laha T, Kaewkes S, Sithithaworn P, Periago MV, Bhudhisawasdi V, Yonglitthipagon P, Mulvenna J, Brindley PJ, Loukas A and Bethony JM: Elevated plasma il-6 associates with increased risk of advanced fibrosis and cholangiocarcinoma in individuals infected by opisthorchis viverrini. PLoS Negl Trop Dis 6(5): e1654, 2012. PMID: 22629477. DOI: 10.1371/journal.pntd.0001654

31 Yang P, Li Z, Li H, Lu Y, Wu H and Li Z: Pyruvate kinase m2 accelerates pro-inflammatory cytokine secretion and cell proliferation induced by lipopolysaccharide in colorectal cancer. Cell Signal 27(7): 1525-1532, 2015. PMID: 25778902. DOI: 10.1016/j.cellsig.2015.02.032

32 Qian Z, Hu W, Lv Z, Liu H, Chen D, Wang Y, Wu J and Zheng $\mathrm{S}$ : Pkm2 upregulation promotes malignancy and indicates poor prognosis for intrahepatic cholangiocarcinoma. Clin Res Hepatol Gastroenterol, 2019. PMID: 31303531. DOI: 10.1016/j.clinre. 2019.06.008

33 Luo W and Semenza GL: Pyruvate kinase $\mathrm{m} 2$ regulates glucose metabolism by functioning as a coactivator for hypoxiainducible factor 1 in cancer cells. Oncotarget 2(7): 551-556, 2011. PMID: 21709315. DOI: 10.18632/oncotarget.299

34 Hsieh YS, Liao CH, Chen WS, Pai JT and Weng MS: Shikonin inhibited migration and invasion of human lung cancer cells via suppression of c-met-mediated epithelial-to-mesenchymal transition. J Cell Biochem 118(12): 4639-4651, 2017. PMID: 28485480. DOI: $10.1002 /$ jcb. 26128

35 Huang C and Hu G: Shikonin suppresses proliferation and induces apoptosis in endometrioid endometrial cancer cells via modulating mir-106b/pten/akt/mtor signaling pathway. Biosci Rep 38(2), 2018. PMID: 29449346. DOI: 10.1042/BSR20171546

36 Guo XP, Zhang XY and Zhang SD: [clinical trial on the effects of shikonin mixture on later stage lung cancer]. Zhong Xi Yi Jie He Za Zhi 11(10): 598-599, 580, 1991. PMID: 1806305.

37 Liang W, Cui J, Zhang K, Xi H, Cai A, Li J, Gao Y, Hu C, Liu Y, Lu Y, Wang N, Wu X, Wei B and Chen L: Shikonin induces ros-based mitochondria-mediated apoptosis in colon cancer. Oncotarget 8(65): 109094-109106, 2017. PMID: 29312593. DOI: $10.18632 /$ oncotarget.22618

38 Zheng H, Huang Q, Huang S, Yang X, Zhu T, Wang W, Wang H, He S, Ji L, Wang Y, Qi X, Liu Z and Lu L: Senescence inducer shikonin ros-dependently suppressed lung cancer progression. Front Pharmacol 9: 519, 2018. PMID: 29875661. DOI: $10.3389 /$ fphar.2018.00519

39 Chang IC, Huang YJ, Chiang TI, Yeh CW and Hsu LS: Shikonin induces apoptosis through reactive oxygen species/extracellular signal-regulated kinase pathway in osteosarcoma cells. Biol Pharm Bull 33(5): 816-824, 2010. PMID: 20460760. DOI: $10.1248 / \mathrm{bpb} .33 .816$

Received June 18, 2020

Revised July 10, 2020

Accepted July 14, 2020 